\title{
Tuning of narrow-bandwidth photonic crystal devices etched in InGaAsP planar waveguides by Liquid Crystal infiltration
}

\author{
Harm H.J.E. Kicken ${ }^{a}$, Ionut Barbu ${ }^{a}$, Sander P. Kersten ${ }^{a}$, Mehmet A. Dündar ${ }^{a}$, Rob W. van \\ der Heijden ${ }^{a}$, Fouad Karouta ${ }^{a}$, Richard Nötzel ${ }^{a}$, Emile van der Drift ${ }^{b}$ and Huub W.M. \\ Salemink ${ }^{b}$ \\ ${ }^{a}$ COBRA Research Institute and Center for NanoMaterials, Eindhoven University of \\ Technology, PO Box 513, NL-5600 MB Eindhoven, The Netherlands. \\ ${ }^{b}$ Kavli Institute of Nanoscience, Delft University of Technology, P.O. Box 5053, NL-2600 GB \\ Delft, The Netherlands.
}

\begin{abstract}
Photonic crystal (PC) devices in the InP/InGaAsP/InP planar waveguide system exhibiting narrow bandwidth features were investigated for use as ultrasmall and tunable building blocks for photonic integrated circuits at the telecom wavelength of $1.55 \mu \mathrm{m}$. The $\mathrm{H} 1$ cavity, consisting of a single PC-hole left unetched, represents the smallest possible cavity in a dielectric material. The tuning of this cavity by temperature was investigated under the conditions as etched and after the holes were infiltrated with liquid crystal (LC), thus separating the contributions of host semiconductor and LC-infill. The shift and tuning by temperature of the MiniStopBand (MSB) in a W3 waveguide, consisting of three rows of holes left unetched, was observed after infiltrating the PC with LC. The samples finally underwent a third processing step of local wet underetching the PC to leave an InGaAsP membrane structure, which was optically assessed through the ridge waveguides that remained after the under etch and by SNOM-probing.
\end{abstract}

\section{INTRODUCTION}

Recently, Photonic crystals ${ }^{1}$ (PCs) have received much attention for applications in photonic integrated circuits (PIC). Part of the attractiveness of PCs is the large range of functionalities for light manipulation, e.g. guiding, localization, dispersion engineering, filters, couplers etc. For PIC applications, operating at telecom wavelengths, a practical implementation of PCs is a triangular array of holes deeply etched into a low vertical index contrast $\mathrm{InP} / \mathrm{InGaAsP} / \mathrm{InP}$ slab waveguide, ${ }^{2}$ i.e. a planar of quasi 2D PC. The field is confined in the out-of-plane direction since the InGaAsP core layer has a slightly higher refractive index than the InP cladding layers. The advantages of this approach are a better structural integrity and heat dissipation as compared to membrane type PCs, particularly for active devices. The use of low vertical index contrast waveguides represents a considerable technological challenge, since very high aspect ratio holes have to be achieved.

Most PC applications require the use of introduced defects to obtain structures with increased or specific functionality. These intended "defects" may be achieved either by lithographic means during fabrication or by post-production methods. The latter methods include infiltration and use of micro-electromechanical systems. The method of post-production tuning is particularly interesting due to the diversity of the possible infills, which may be both passive and active materials. Recently, a number of experiments have been performed using liquid crystals (LC), ${ }^{3,4}$ several types of polymers ${ }^{5-7}$ and chalcogenide glasses. ${ }^{8}$ An added advantage of these tuning methods is the possibility of quick and reversible prototyping in the sense of breadboard-type devices. ${ }^{9,10}$

Many PIC components such as low-threshold lasers ${ }^{11,12}$ or drop filters ${ }^{13}$ require sharp spectral features with minimum component-size. Since the smallest obtainable mode volume with dielectrics is of the order of the cube wavelength, the H1 cavity, i.e. size of one unit cell in a PC, seems a natural device choice. H1 cavities or modifications thereof, ${ }^{14}$ were only reported in high vertical refractive index contrast systems such as suspended membranes in air and for AlGaAs/GaAs half membrane structures ${ }^{15}$ so far.

E-mail: h.h.j.e.kicken@tue.nl

Photonic and Phononic Crystal Materials and Devices IX,

edited by Ali Adibi, Shawn-Yu Lin, Axel Scherer, Proc. of SPIE Vol. 7223,

72230C · @ 2009 SPIE - CCC code: 0277-786X/09/\$18 - doi: 10.1117/12.808403

Proc. of SPIE Vol. $722372230 \mathrm{C}-1$ 
Other relevant PIC components include the PC waveguide. It is not only useful for transport of light but it also has large potential for devices functionalities, such as wavelength division demultiplexing. ${ }^{16}$ The W3 (three rows of holes left unetched) PC waveguide is of particular interest since it is the smallest PC waveguide which displays a mini-stopband (MSB) in the center of the band gap. ${ }^{17}$

In this work we investigate by transmission spectroscopy the properties of the $\mathrm{H} 1$ cavity and the W3 PC waveguide in the deeply etched $\mathrm{InP} / \mathrm{InGaAsP} / \mathrm{InP}$ system before and after infiltration with LC. Subsequently, the spectral features of both devices are tuned by variation of the temperature, exploiting the large temperature dependence of the LC refractive index. Though the W3 PC waveguide and its properties has been reported for the InP/InGaAsP/InP system, tuning of the MSB feature has not been reported.

Scanning near-field microscopy (SNOM) and infra-red microscopy is used to image the scattered and evanescent fields after wet-chemical underetching of the deeply etched devices.

\section{EXPERIMENTAL}

PCs were fabricated by etching a triangular lattice of holes into an InP/InGaAsP/InP slab waveguide, consisting of a $0.5 \mu \mathrm{m}$ InP cladding layer on top of $0.5 \mu \mathrm{m}$ InGaAsP core layer on an InP substrate. The core layer has a refractive index of 3.35 and the cladding layers have refractive index of 3.17. The etching of the approximately $2.5 \mu \mathrm{m}$ deep holes, with $200 \mathrm{~nm}$ diameter was achieved with $\mathrm{Cl}_{2} / \mathrm{O}_{2}$ chemistry using inductively coupled plasma (ICP) reactive ion etching (RIE), see figure 1. The air filling fraction, $f$, of the holes was designed to be 0.3. The SEM picture displays a cross-section of a typical device used for transmission spectroscopy.

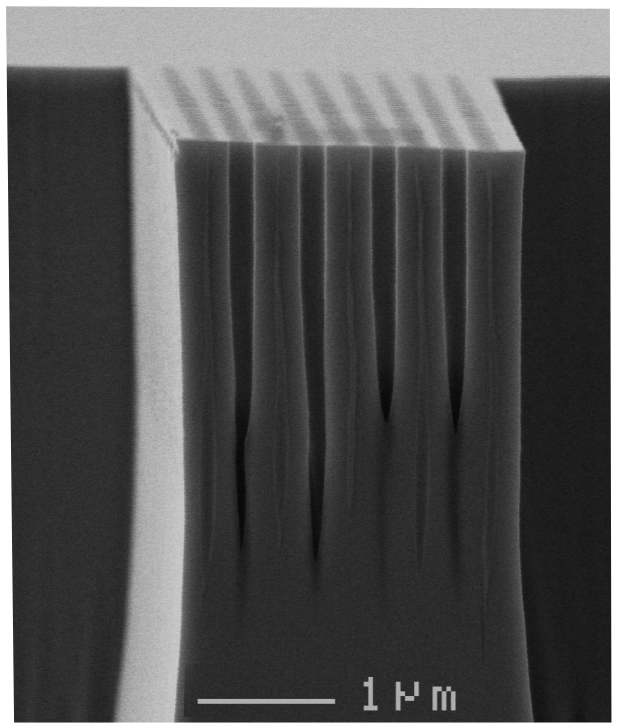

Figure 1. SEM picture of the cross-section of the holes in a PC. The structure shown has a lattice constant of $337 \mathrm{~nm}$. The holes have a diameter of $200 \mathrm{~nm}$ and a depth of $\sim 2.5 \mu \mathrm{m}$.

For the fabrication of the $2.5 \mu \mathrm{m}$ wide access ridge waveguides (RWGs), two processes were employed. In one RWGs were etched in the same process as the PC holes. In the other RWGs were produced in a two step process, in which alignment markers were etched in the same step as the holes. In the next step the holes are covered with a new $400 \mathrm{~nm}$ thick $S i N_{x}$ hard mask layer. Due to the isotropic nature of the $S_{i} N_{x}$ deposition process, the holes are not filled with the $S i N_{x}$, but a nearly flat layer is formed, covering the holes. Since the markers are of considerable size, they remain visible also after application of a second electron beam resist layer. With this two-step process waveguides and PCs can be etched using different processes. The process for etching holes yields very high ridges and generally is not optimum for low RWG losses. Using electron beam lithography for the second step results in good alignment accuracy $(<100 \mathrm{~nm})$ with respect to the PC. In principle the second step could be partly replaced by optical lithography, to reduce electron beam exposure time. 
Transmission spectroscopy was carried out using an end-fire technique using a tunable laser in the range of $1470 \mathrm{~nm}$ to $1570 \mathrm{~nm}$ in TE polarization.

Several structures were fabricated by leaving holes unetched. These include the H1 (a single unetched hole) and W3 (three rows unetched) waveguide. After PC and waveguide fabrication, the devices were first optically characterized. As a next step, the PCs were infiltrated with the liquid crystal (LC) K15 (5CB). This LC has a crystalline to nematic transition temperature, $T_{c n}$, of $23^{\circ} \mathrm{C}$ and a nematic to isotropic transition or clearing temperature, $T_{n i}$, of $35.4^{\circ} \mathrm{C}$. In the nematic state the LC-K15 is birefringent with $n_{o}=1.516$ and $n_{e}=1.682$ as the ordinary (electric field perpendicular to the average orientation of the LCs) and extraordinary refractive indices respectively, at a wavelength of $1.5 \mu \mathrm{m}$. In the isotropic state, the refractive index at $\mathrm{T}=40^{\circ} \mathrm{C}$ is $n_{i}=$ 1.575. Exploiting the relatively large change of the refractive index due to the nematic to isotropic transition, the spectra of the defect structures were tuned.

Transmission spectroscopy is carried out on both the H1 cavity and the W3 PC waveguide devices before and after infiltration and as a function of temperature. Measurement of the unfilled devices at elevated temperature is important to quantify the change of refractive index with temperature of the semiconductor layer stack. In order to determine the infiltrated fraction of the holes (infiltration efficiency), the devices were measured after filling, at a temperature above $T_{n i}$. Above $T_{n i}$ the refractive index may be assumed equal to the isotropic value, $n_{i}$, thus the infiltration efficiency may be determined by fitting the spectral features of the measured spectrum with 2D-Finite Difference Time Domain (FDTD) simulations.

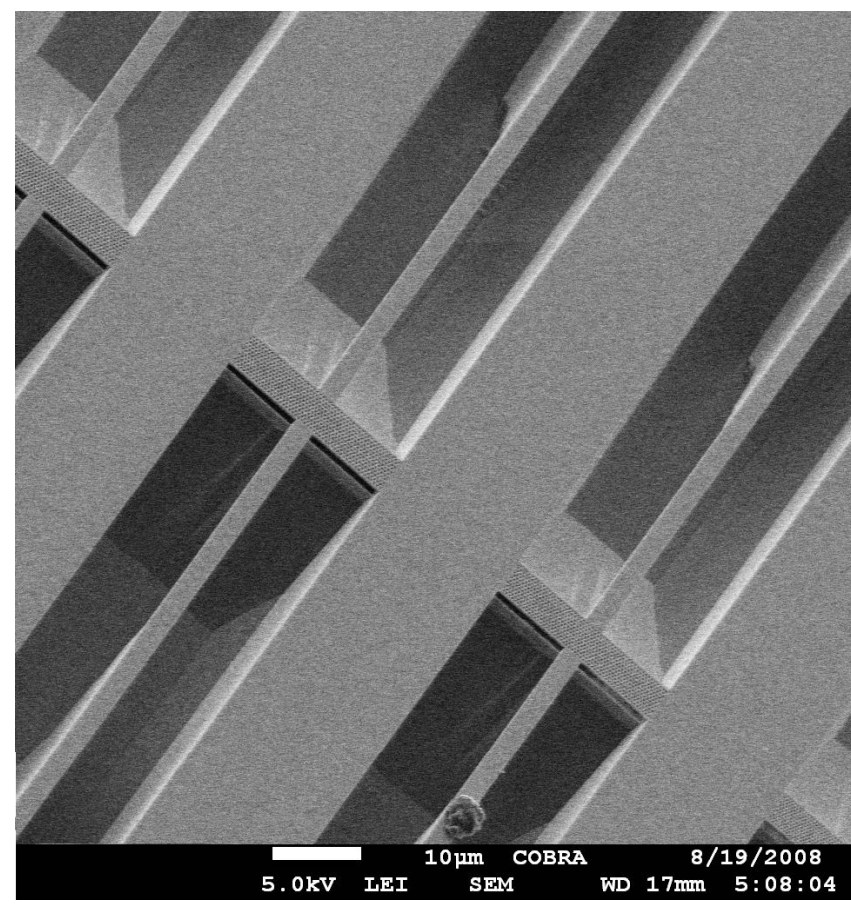

InGaAsP

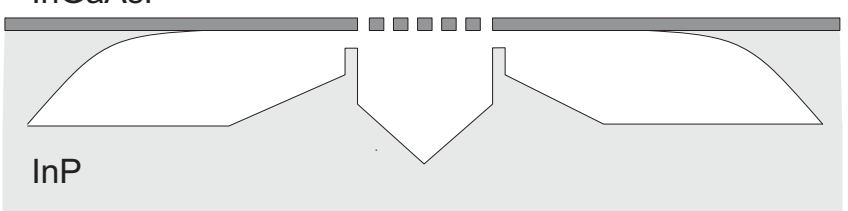

(b)

(a)

Figure 2. a) SEM picture of PC structures and access waveguides after selectively etching away the cladding layers, leaving an InGaAsP membrane. The PC is completely underetched, the RWGs are only partially underetched close to both the cleaved sample edges and the PC. The underetch near the sample edges can be removed by another cleave step. b) sketch of the cross-section along a waveguide.

In order to combine membrane devices, which generally have sharper spectral features than deeply etched devices, with conventional RWGs, the cladding layers were chemically etched away using a solution of $\mathrm{HCl}$ in water (1:4), leaving an InGaAsP suspended membrane. Despite the $10 \mu \mathrm{m}$ deep trenches and narrow RWGs, the RWGs were not completely underetched, except for approximately $50 \mu \mathrm{m}$ stretches near to the cleaved 
sample edges as well as close to the PC, see figure 2. Underneath the PC the SEM picture shows a trapezoid structure, however, the PC was verified to be suspended above this trapezoidal structure by careful inspection under different angles and of broken structures. A qualitative sketch of the cross-section along the waveguide, based on SEM data is given in figure 2(b). The precise shape of the underetch, caused by peculiarities of the anisotropic InP wet etching is not relevant for the performance of the membrane and is not analyzed further.

Prior to infiltration, the samples were chemically treated to improve the wetting angle, an important parameter for infiltration succes. First an oxygen plasma was employed to clean the surface from possible organic dust particles accumulated on the surface. Subsequently, the surface oxides were removed using a solution of $10 \%$ phosphoric acid in water and the sample was thereafter rinsed with isopropanol which improves the wetting by the LC. In order to increase the infiltration efficiency both the LC and sample were introduced into an evacuated chamber. This practice makes sure that no air is trapped inside the air-holes and no air-bubbles remain in the LC. The LC was heated above the clearing temperature and deposited onto the surface as a large droplet.

\section{RESULTS}

\subsection{H1 cavity}

The transmission spectrum of the H1 (SEM detail in figure 3a)) resonance is shown in figure 3c) curve 1. The bold line indicates a Lorentzian fit of the resonance envelope, yielding a Q-factor of 65. Due to the presence of the access RWGs a large beat and fine Fabry-Perot (FP) fringes (resolved in figure 3b)) are observed. These FP fringes correspond to the approximately $1 \mathrm{~mm}$ long FP cavities formed in the access RWGs between the cleaved end facets and the PC. The beat is due to the length difference of the entrance and exit waveguide, on the order of $50 \mu \mathrm{m}$.

So far, result for the H1 cavity in the InP-system have only been reported for membranes with relatively high filling factors. The Q-factor found for our deeply etched device is similar to values found for membrane devices. For InP membranes a Q-factor of 70 for $\mathrm{f}=0.43$ and $\mathrm{Q}=100$ for $\mathrm{f}=0.55^{18}$ is obtained. For GaAs-based devices higher values are found, respectively 270 and 250 for AlGaAs/GaAs half-membranes ${ }^{15}$ and GaAs membranes. ${ }^{19}$ For membrane devices generally much higher values are found after slight rearrangement of the H1 cavity nearest neighbor holes. ${ }^{20}$

Theoretically, the $\mathrm{H} 1$ defect cavity displays a single degenerate resonance in the middle of the bandgap, for moderately low filling factors $(<0.38){ }^{18,21}$ The mode in figure 3 therefore corresponds to the dipole mode. This dipole mode is known to have a relatively low Q-factor. ${ }^{22}$ In applications where very high Q-factors are important, the hexapole mode, which is only available for high filling factors or after rearrangement of holes, ${ }^{23}$ is often used.

To obtain an estimate for the reflectivity of the PC mirrors, the expression for a one-dimensional FP-type cavity with one row of missing holes will be used. The FP cavity is only confined in one dimension, while the H1 cavity is confined in two dimensions, therefore the H1 cavity is expected to yield higher losses. This analysis will thus yield a lower bound on the reflectivity. The reflectivity can be calculated from $Q=m F=m \pi \sqrt{R} /(1-R)$, with $m=3$, the mode number associated with the cavity length, $F$ the finesse and $R$ the reflectivity. The mode number is taken as 3 times the lattice constant, consistent with FP-type cavities. ${ }^{24}$ This number, which seems rather high, is due to the penetration depth of the electric field into the PC cladding. From this, the reflectivity, $\mathrm{R}$, is estimated to be approximately 0.85 .

The measured Q-factor compares favorably to previously reported results of FP-type cavities in deeply etched InGaAsP, consisting of a PC with one row of missing holes, where Q-factors of 20 to 30 were found. ${ }^{2}$ More recently, however, optimization in etching (Chemically Assisted Ion Beam Etching (CAIBE)) resulted in an increase of the Q-factor for these FP-type defect cavities to approximately $300 .^{3,25}$

Given that improved etching for FP-cavities, as mentioned above, has led to a dramatic increase of the Qfactor, it is likely that a similar improvement of the Q-factor may be possible for H1-cavities in these deeply etched systems.

After infiltration with LC the spectrum of the H1 cavity was again measured, see figure 3(c), curve 2. A large frequency shift of $\Delta(a / \lambda)=8 \cdot 10^{-3}$ was observed compared to the uninfiltrated case (figure $3(\mathrm{c}$ ), curve 


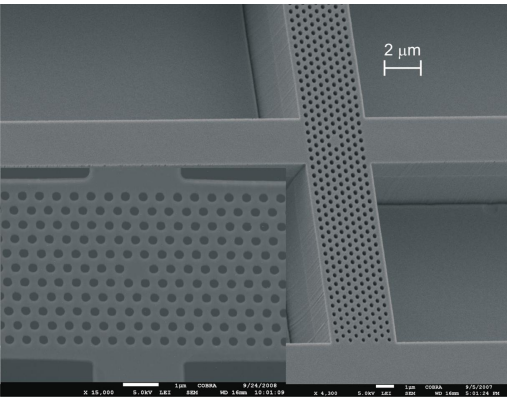

(a)

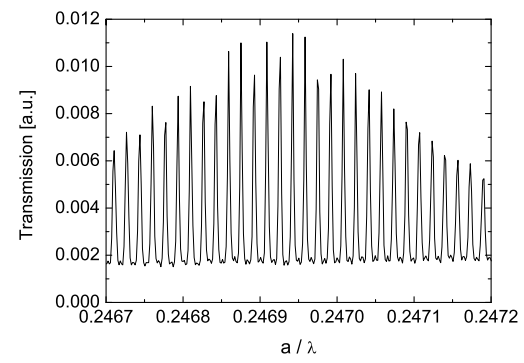

(b)

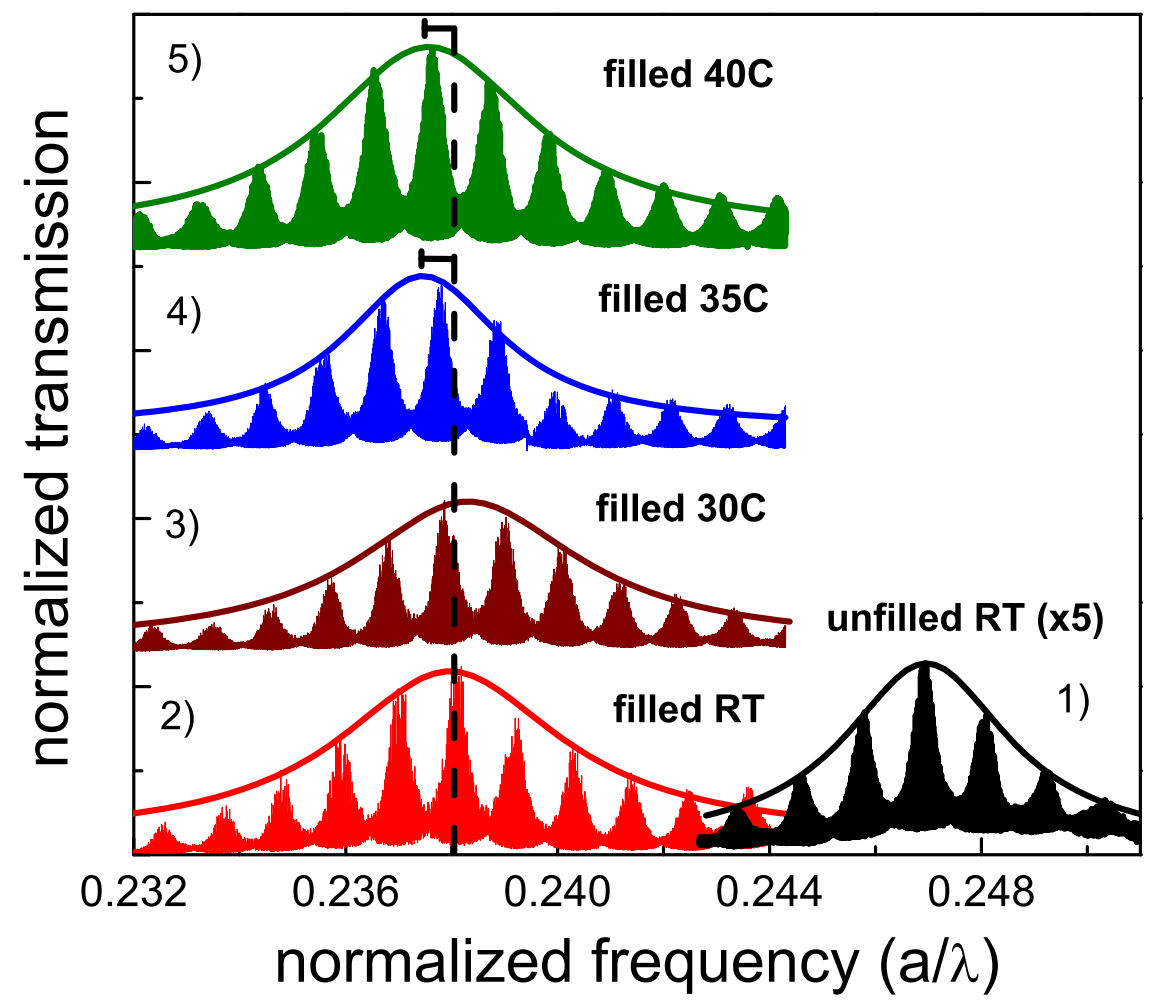

(c)

Figure 3. Temperature tuning of the H1 resonance. Figure 3(a) shows a SEM image of the H1 defect cavity, in the center of the cross formed by the access RWGs and the PC, further detailed in the inset. In figure 3(b) the fine FP fringes are shown arising from the cavities formed by the RWGs. In subfigure c), curve 1 through 5 the spectrum of the H1 resonance is shown. Unfilled at RT (1), filled with LC (2) and at three elevated temperatures, $30^{\circ} \mathrm{C}(3)$, with the LC still in the nematic state, $35^{\circ} \mathrm{C}(4)$ near the clearing temperature, $40^{\circ} \mathrm{C}(5)$, in the isotropic state.

1). The Q-factor decreased as a result of the infiltration to 35. Also, a considerable increase of the transmission level with a factor of 5 was found. This is attributed to the increased coupling to the incoming waveguide mode. As a result of the decrease of the refractive index contrast the confinement is reduced and the modal pattern dramatically expands, giving rise to a larger modal overlap with the waveguide, see figure 4.

To demonstrate the feasibility of tuning, the temperature was varied across the nematic to isotropic transition of the LC, which results in a step-like change of refractive index of the LC. Figure 3(c), curve 2 through 5 show the resonance at four relevant temperatures. At room temperature (RT) (1) the LC is in the nematic state, just above the transition from the crystalline state. From $30^{\circ} \mathrm{C}(3)$ to $40^{\circ} \mathrm{C}(5)$ the $\mathrm{LC}$ is driven from the nematic state to isotropic state, with the measurement at $35^{\circ} \mathrm{C}$ (4) being close to the clearing temperature. Taking the nematic state (RT) as a reference (black vertical line), a redshift of $7 \pm 1 \mathrm{~nm}$ is consistently observed when crossing the clearing temperature. Note that increasing the temperature from $35^{\circ} \mathrm{C}$ to $40^{\circ} \mathrm{C}$ shows no shift, indicating that the $\mathrm{LC}$ is in the isotropic state. A comparable redshift of the resonance frequency was also found for FP-type cavities. ${ }^{4}$ For empty cavities a redshift of $3 \mathrm{~nm}$ is found for a temperature variation from RT to $40^{\circ} \mathrm{C}$, in agreement with the expected refractive index variation of InGaAsP.

The LC filling efficiency can be found by fitting the $40^{\circ} \mathrm{C}$ spectrum with $2 \mathrm{D}$ FDTD calculations with the effective refractive index of the slab waveguide, since the refractive index of the LC in the isotropic state is known. This fit yields a filling efficiency of 0.6. Combining this value with the 2D-FDTD fits of the $30^{\circ} \mathrm{C}$ (LC nematic state) spectrum yields a refractive index of 1.5 , indistinguishable from the ordinary index, $n_{o}$, suggesting 


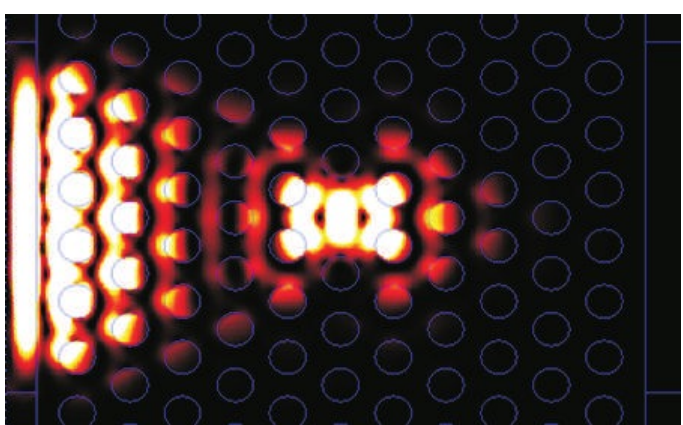

(a)

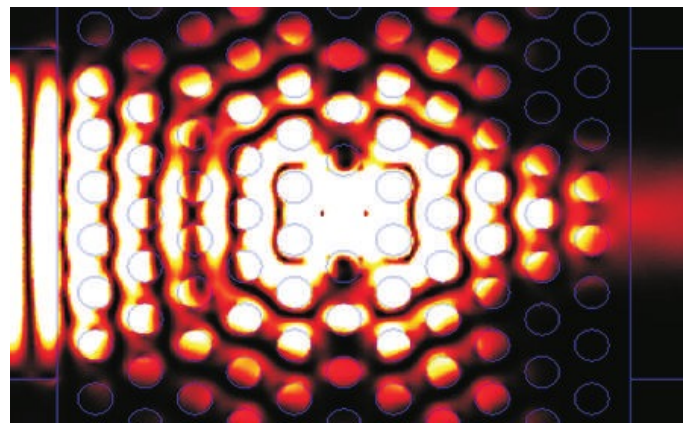

(b)

Figure 4. Intensity plots obtained by $2 \mathrm{D}$ FD calculations showing the $\mathrm{H} 1$ cavity dipole mode at resonance and the connecting RWG on the left. a) displays the intensity pattern for air filled holes, b) shows the pattern for LC filled holes.

a director field orientation near-parallel to the hole axis. However, given the large uncertainties strong claims with respect to the orientation cannot be made.

\subsection{W3 PC waveguide}

The W3 PC waveguide is an important device since it displays a sharp MSB in the center of the bandgap. The MSB originates from coupling between the fundamental mode and a higher order counter-propagating mode. ${ }^{17}$ This coupling effect generally occurs when the dispersion curves of modes of equal parity cross, and produce an anti-crossing behavior. ${ }^{16,17}$ In the case of the W3 waveguide the fundamental mode is even and will thus only couple to the even, higher order mode. The anticrossing leads to a minigap in the waveguide modes, which is visible as a narrow dip in the transmission spectrum. Due to this coupling effect, the group velocity of the modes directly near the anti-crossing frequency is very low. The propagation occurs in a FP-like mode, bouncing between the PC mirrors. These modes have a strong interaction with the PC, thus yielding high losses. The total width of the MSB dip is a combination of the stopband and increased losses adjacent to the stopband.

In figure 5(a) the transmission spectrum of a W3 PC waveguide with MSB is shown before (black line) and after (grey line) infiltration. The inset displays a SEM micrograph of the device. The features present in the trenches defining the tapered waveguides are due to improper implementation of proximity effect corrections of the electron beam lithography process, and should be ignored. The RWGs are tapered to match the size of the PC waveguide to reduce coupling loss. Both before and after infiltration the sharp transmission dip, indicating the MSB is observed. As a result of the LC infiltration in the holes the MSB feature is red-shifted by $30.3 \mathrm{~nm}$. Also, the overall transmission is increased by a factor of 2 , which is attributed to reduced out-of-plane losses of the fundamental mode.

After the LC infiltration the shift of the MSB feature was investigated with respect to the temperature at five relevant temperatures. Close to the crystalline to nematic transition (RT), in the nematic state $\left(30^{\circ} \mathrm{C}\right)$, near the clearing temperature $\left(35^{\circ} \mathrm{C}\right)$ and well into the isotropic state at $40^{\circ} \mathrm{C}$ and $45^{\circ} \mathrm{C}$. As the temperature is raised from RT to $30^{\circ} \mathrm{C}$ no shift is found. Raising the temperature further, to $35^{\circ} \mathrm{C}$, a $8 \mathrm{~nm}$ redshift of the MSB feature is observed, associated with the refractive index change when crossing the clearing temperature. Further increase of the temperature does not lead to an additional shift.

Due to the temperature change the refractive index of the host semiconductor is also changed. However, since this effect is gradual and is estimated to be around $3 \mathrm{~nm}$ over the whole temperature range, it can be concluded that the observed shift is primarily caused by the refractive index change of the LC.

By fitting the measured spectrum at $40^{\circ} \mathrm{C}$ and assuming an isotropic refractive index of 1.575 the filling efficiency is found to be approximately 0.8 . This value is substantially higher than found for the H1-cavity $(\sim$ 0.6). As both devices were fabricated on the same sample the filling efficiencies were expected to be similar, however, it is known that the wetting properties of the LC depend critically on the exact chemical state of the surface, which may vary across the sample. 


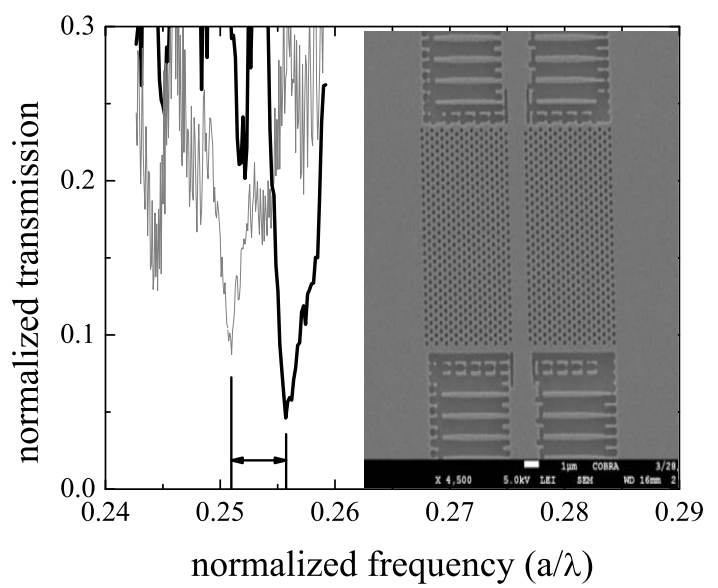

(a)

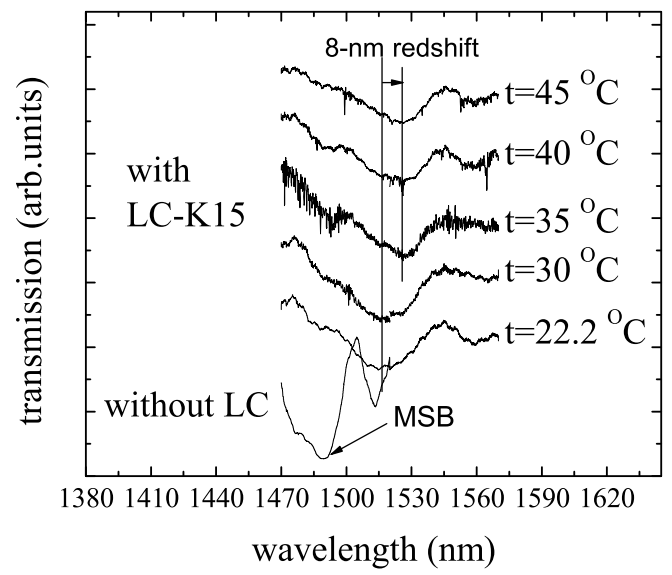

(b)

Figure 5. 5(a) Spectrum of a W3 PC waveguide before (black line) and after (gray line) infiltration with LC K15. The inset displays a SEM micrograph of the actual structure used. 5(b) The behavior of the W3 MSB as a result of LC infiltration and subsequent temperature tuning.

Fitting the MSB feature using 2D-FDTD simulation at RT, with the LC in its nematic state, yields a refractive index of 1.37. Taking the filling efficiency into account a nematic refractive index of 1.5, indistinguishable from $n_{o}$, is found, consistent with the findings for the H1-cavity. Although no strong claims can be made due to the large uncertainties, this suggest a LC director field perpendicular to the hole axis in the nematic state.

\subsection{Microscopy}

Infrared microscopy and SNOM measurements were carried out on deeply etched samples that underwent a wet chemical underetch, removing the InP claddings, to realize an InGaAsP membrane PC (see figure 2). Note that the RWGs are not completely underetched, remaining functional for transmission spectroscopy. Since these devices were fabricated as deeply etched devices the resulting membrane is no longer single mode with respect to the thickness. Thick membranes are usually avoided since the bandgap is closed due to the appearance of a second order guided mode. Recently, however, it was shown that thick membranes are useful for high-Q cavities. $^{26}$

For the present experiment, a deeply etched sample which did not show photonic band features due to poor hole quality was selected for underetching. This will eliminate the influence of the possible poor quality of the deep parts of the holes. After the underetching the airband edge was recovered (see figure 7(d), proving that the quality of the cladding layer is of the utmost importance in deeply etched structures. The dielectric edge was not available in the lithotuned structures. The data illustrate that membrane devices can be easily combined with conventional RWGs standard PIC. The properties of PCs may dramatically improve after underetching.

In order to have an indication on the light scattered by the underetched structure particularly at the transition from ridge to suspended membrane, infra-red images were taken with a microscope objective (see figure 6). Subfigures b) and d) show a visible light image of the structure in two focal planes. In image d) the focus is on the top of the RWG, while b) shows the focus on the bottom of the trenches, showing clearly the partially underetched RWG. Subfigure a) and c) display the infra-red scattered light from the structure in the air band and band gap respectively. In dashed lines the topography of the sample is highlighted for clarity. Note that the light is coupled in from the top RWG. The patterns as indicated by subfigures a) and c) exhibit the same features. They indicate a considerable scattering at the transition from bridge-type waveguide to RWG, despite that the wet etching leads to a natural smooth transition, see figure 2(b). In addition strong scattering is seen from the PC device itself. Also, quite a large portion of the light apparently is scattered by the PC in almost perpendicular directions, exiting the sample at the next device structure. 


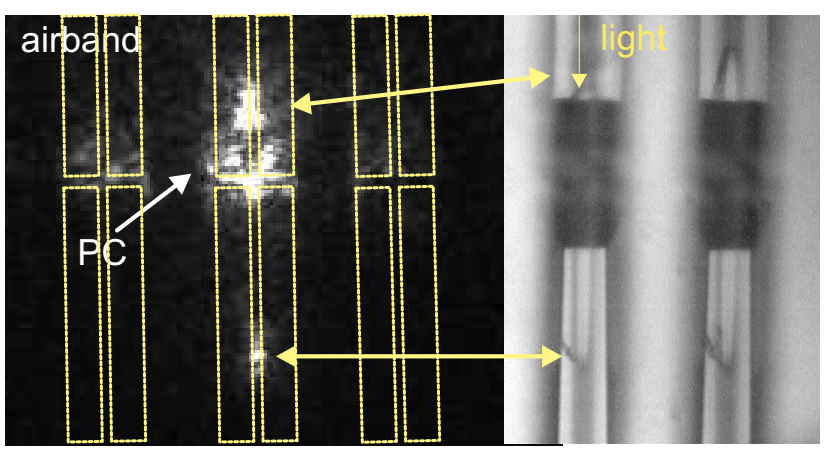

a

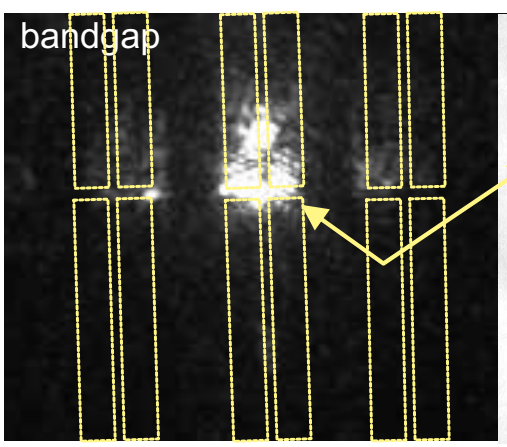

C

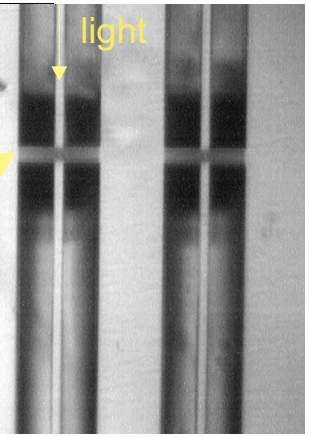

d

Figure 6. Infra-red and visible images of a PC device. Visible light images b) and d) are taken at different focal planes, respectively with the focus on the $\mathrm{PC}$ and the bottom of the trenches, showing the extent of the underetching. Infra-red images a) and c) show the location and intensity of scattering center in the airband and bandgap respectively.

The reflective properties of the PC are emphasized when comparing the air-band image (a) to the bandgap image (c). In the band gap the light exiting the structure after the PC is severely reduced, while light outcoupling is increased from the other scattering locations.

Before underetching, no light could be measured in the SNOM setup due to the thick cladding, strongly reducing evanescent fields. Figure 7 shows the result obtained by SNOM measurements after underetching. Subfigure a) schematically indicates the measurement configuration. An atomic force microscopy image shows both the orientation and the location of the SNOM pictures b) and c). The light is coupled in from the lower left side.

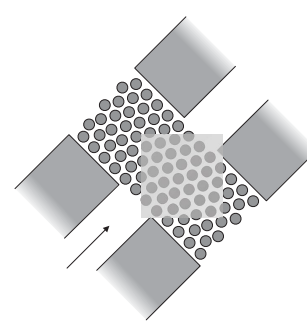

(a)

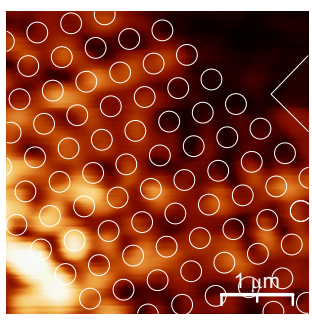

(b)

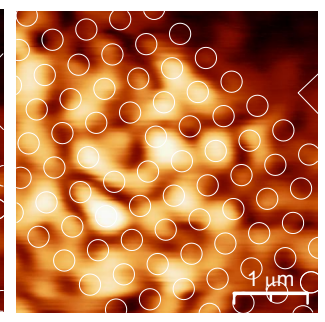

(c)

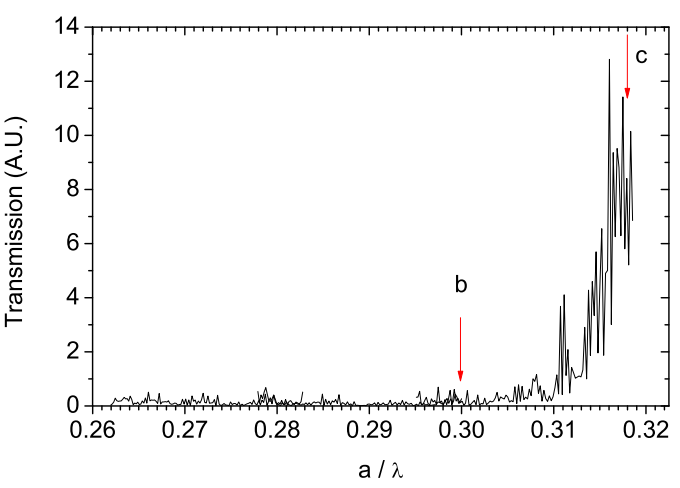

(d)

Figure 7. Transmission spectra and corresponding SNOM images. Subfigure a) details the measurement configuration in which images b) and c) were taken. Subfigures d) and e) are the transmission spectra before and after underetching. Images b) and c) are taken at the frequency indicated in graph e).

Figure 7(d) displays part of the transmission spectrum measured, detailing the airband edge. Arrows indicate the frequencies at which the SNOM images in subfigures b) and c) were taken. Although scattered fields probably are responsible for a considerable part of the collected light, the pictures clearly show the light penetrating the $\mathrm{PC}$ to a larger extent when a frequency in the airband is selected with respect to a frequency in the bandgap.

\section{CONCLUSIONS}

In summary, an $\mathrm{H} 1$ cavity was fabricated in the deeply etched InP-system with a Q-factor of 65, comparable to FP-type cavities with PC holes of comparable quality. The cavity resonance was red-shifted by $\Delta(a / \lambda)=8 \cdot 10^{-3}$ 
$\mathrm{nm}$ using LC infiltration, while the Q-factor was reduced with a factor of two. The infiltration efficiency was determined to be 0.6. Subsequent temperature tuning of the LC refractive index exhibited a further discrete shift $8 \pm 1 \mathrm{~nm}$ red-shift upon crossing the LC clearing temperature. On the same sample a W3 PC waveguide was produced exhibiting a MSB. The MSB feature was redshifted by LC infiltration by $30 \pm 1 \mathrm{~nm}$, with an infiltration efficiency of 0.8. Temperature tuning experiments show a sudden $7 \pm 1 \mathrm{~nm}$ redshift at the nematic to isotropic transition temperature, consistent with the $\mathrm{H} 1$ cavity. The nematic refractive index of the LC in both devices is found to be indistinguishable from the ordinary index, approximately 1.5. Underetching of deeply etched device may be used to improve spectral features, since deeply etched devices display a large dependence on the quality of the holes in the claddings, removing the claddings result in membrane devices of reasonable quality.

\section{ACKNOWLEDGMENTS}

The authors gratefully acknowledge useful discussions with C.W.M. Bastiaansen and D.J. Broer and thank P. Nouwens for contributions to the experimental work. This work is part of the research programme of the 'Stichting voor Fundamenteel Onderzoek der Materie (FOM)', which is financially supported by the 'Nederlandse Organisatie voor Wetenschappelijk Onderzoek (NWO)'. Part of this research is supported by NanoNed, a technology programme of the Dutch ministry of Economic Affairs via the foundation STW.

\section{REFERENCES}

1. J. Joannopoulos, R. Meade, and J. Winn, Photonic crystals: molding the flow of light, Princeton Univ. Press, Princeton, NJ, 2008.

2. R. Ferrini, D. Leuenberger, M. Mulot, J. M. M. Qiu, M. Kamp, A. Forchel, S. Anand, and R. Houdré, "Optical study of two-dimensional InP-based photonic crystals by internal light source technique," J. Quantum Elec. 38, p. 786, 2002.

3. J. Martz, R. Ferrini, F. Nesch, L. Zuppiroli, B. Wild, L. Dunbar, R. Houdré, M. Mulot, and S. Anand, "Liquid crystal infiltration of InP-based photonic crystals," J. Appl. Phys. 99, p. 103105, 2006.

4. R. Ferrini, J. Martz, L. Zuppiroli, B. Wild, V. Zabelin, L. Dunbar, R. Houdré, M. Mulot, and S. Anand, "Planar photonic crystals infiltrated with liquid crystals: optical characterization of molecule orientation," Opt. Lett. 31, p. 1238, 2006.

5. R. van der Heijden, C. Carlström, J. Snijders, R. van der Heijden, F. Karouta, R. Nötzel, H. Salemink, B. Kjellander, C. Bastiaansen, D. Broer, and E. van der Drift, "InP-based two-dimensional photonic crystals filled with polymers," Appl. Phys. Lett. 88, p. 161112, 2006.

6. P. El-Kallassi, R. Ferrini, L. Zuppiroli, N. L. Thomas, R. Houdré, A. Berrier, S. Anand, and A. Talneau, "Optical tuning of planar photonic crystals infiltrated with organic molecules," J. Opt. Soc. Am. B 24, p. $2165,2007$.

7. S. Tay, J. Thomas, B. Momeni, M. Askari, A. Adibi, P. Hotchkiss, S. Jones, S. Marder, R. Norwood, and N. Peyghambarian, "Planar photonic crystals infiltrated with nanoparticle/polymer composites," Appl. Phys. Lett. 91, p. 221109, 2007.

8. A. Faraon, D. Englund, D. Bulla, B. Luther-Davies, B. Eggleton, N. Stoltz, P. Petroff, and J. Vuĉković, "Local tuning of photonic crystal cavities using chalcogenide glasses," Appl. Phys. Lett. 92, p. 043123, 2008.

9. F. Intonti, S. Vignolini, V. Türck, M. Colocci, P. Benotti, L. Pavesi, S. Schweizer, R. Wehrspohn, and D. Wiersma, "Rewritable photonic circuits," Appl. Phys. Lett. 89, p. 211117, 2006.

10. C. Smith, U. Bog, S. Tomljenovic-Hanic, M. Lee, D. Wu, L. O'Faolain, C. Monat, C. Grillet, T. Krauss, C. Karnutsch, R. McPhedran, and B. Eggleton, "Reconfigurable microfluidic photonic crystal slab cavities," Opt. Express 16, p. 15887, 2008.

11. O. Painter, R. Lee, A. Scherer, A. Yariv, J. O’Brien, P. Dapkus, and I. Kim, "Two-dimensional photonic band-gap defect mode laser," Science 284, p. 1819, 1999.

12. C. Monat, C. Seassal, X. Letarte, P. Viktorovitch, P. Regreny, M. Gendry, P. Rojo-Romeo, G. Hollinger, E. Jalaguier, S. Pocas, and B. Aspar, "InP 2D photonic crystal microlasers on silicon wafer: room temperature operation at $1.55 \mu \mathrm{m}, "$ Electron. Lett. 37, p. 764, 2001.

13. H. Takano, Y. Akahane, T. Asano, and S. Noda, "In-plane-type channel drop filter in a two-dimensional photonic crystal slab," Appl. Phys. Lett. 84, p. 2226, 2004. 
14. B.-S. Song, S. Noda, T. Asano, and Y. Akahane, "Ultra-high-Q photonic double-heterostructure nanocavity," Nature Mat. 4, p. 207, 2005.

15. A. Kress, F. Hofbauer, N. Reinelt, H. Krenner, M. Bichler, D. Schuh, R. Meyer, G. Abstreiter, and J. Finley, "Investigation of cavity modes and direct observation of Purcell enhancement in 2D photonic crystal defect microcavities," Physica E 26, p. 351, 2005.

16. L. Martinelli, H. Benisty, O. Khayam, G.-H. Duan, H. Heidrich, and K. Janiak, "Analysis and optimization of compact demultiplexer monitor based on photonic-crystal waveguide," J. Lightwave Technol. 25, p. 2385, 2007.

17. S. Olivier, M. Rattier, H. Benisty, C. Weisbuch, C. Smith, R. De La Rue, T. Krauss, U.Oesterle, and R. Houdré, "Mini-stopbands of a one-dimensional system: The channel waveguide in a two-dimensional photonic crystal," Phys. Rev. B 63, p. 113311, 2001.

18. C. Monat, C. Seassal, X. Letrarte, P. Regreny, M. Gendry, P. Rojo Romeo, P. Viktorovitch, M. Le Vassor d'Yerville, D. Cassagne, J. Albert, E. Jalaguier, S. Pocas, and B. Aspar, "Two-dimensional hexagonal-shaped microcavities formed in a two-dimensional photonic crystal on an InP membrane," J. Appl. Phys. 93, p. 23, 2003.

19. C. Reese, B. Gayral, B. Gerardot, A. Imamoğlu, P. Petroff, and E. Hu, "High-Q photonic crystal microcavities fabricated in a thin GaAs membrane," J. Vac. Sci. Technol. B 19, p. 2749, 2001.

20. T. Tanabe, A. Shinya, E. Kuramochi, S. Kondo, H. Taniyama, and M. Notomi, "Single point defect photonic crystal nanocavity with ultrahigh quality factor achieved by using hexapole mode," Appl. Phys. Lett. 91, p. $021110,2007$.

21. O. Painter, J. J. Vuĉković, and A. Scherer, "Defect modes of a two-dimensional photonic crystal in an optically thin dielectric slab," J. Opt. Soc. Am. B 16, p. 275, 1999.

22. W. Zheng, G. Ren, X. Ma, X. Cai, L. Chen, K. Nozaki, and T. Baba, "Dipole mode photonic crystal point defect laser on InGaAsP/InP," J. Crystal Growth 292, p. 341, 2006.

23. S.-K. Kim, G.-K. Kim, S.-H. Kim, Y.-H. Lee, S.-B. Kim, and I. Kim, "Loss management using parityselective barriers for single-mode single-cell photonic crystal resonators," Appl. Phys. Lett. 88, p. 161119, 2006.

24. M. Rattier, H. Benisty, C. Smith, A. Béraud, D. Cassagne, C. Jouanin, T. Krauss, and C. Weisbuch, "Performance of waveguide-based two-dimensional photonic-crystal mirrors studied with FabryPérot resonators," J. Quantum Elec. 37, p. 237, 2001.

25. A. Berrier, M. Mulot, S. Anand, A. Talneau, R. Ferrini, and R. Houdré, "Characterization of the feature-size dependence in $\mathrm{Ar} / \mathrm{Cl} 2$ chemically assisted ion beam etching of InP-based photonic crystal devices," J. Vac. Sci. Technol. B 25, p. 1, 2007.

26. A. Tandaechanurat, S. Iwamoto, M. Nomura, N. Kumagai, and Y. Arakawa, "Increase of Q-factor in photonic crystal H1-defect nanocavities after closing of photonic bandgap with optimal slab thickness," Opt. Express 16, p. 448, 2008. 